\section{POS0401 \\ THE APPLICATION OF MTX-LOADING DNA TETRAHEDRON IN TREATING COLLAGEN-INDUCED ARTHRITIS MICE VIA REGULATING MACROPHAGE}

X. Ge ${ }^{1}$, Y. $\mathrm{Jin}^{1}$, Z. $\mathrm{Mao}^{2}$, Y. Guo ${ }^{2}$, Z. Gu${ }^{1} .{ }^{1}$ Affiliated Hospital of Nantong University, Department of Rheumatology, Nantong, China; ${ }^{2}$ Affiliated Hospital of Nantong University, Research Center of Clinical Medicine, Nantong, China

Background: Rheumatoid arthritis (RA) is a systemic autoimmune disease which mainly affect joints. [1]. Macrophages often infiltrate in the inflammatory joints. Activated macrophages release TNF- $\alpha$, IL-1 $\beta$ to accelerate tissue damage, is one of the most important targets for RA intervention. The traditional drugs currently used commonly have some disadvantages cannot be bypassed[2], while DNA nanostructure is a new type of drugs have precise design, and likewise takes biological effect together[3]. We synthesized a DNA tetrahedron loaded with MTX and conjugated with $\mathrm{HA}$ which targeted to macrophage.

Objectives: To verify whether MTX-loading DNA tetrahedron can regulate the apoptosis and polarization of macrophage and finally improve the condition of CIA model mice by while decrease the side effect of MTX.

Methods: DNA TET was synthesized by mixing signal strand DNA in TM buffer and heated to $95^{\circ} \mathrm{C}$ for $10 \mathrm{~min}$, then cooling to $4{ }^{\circ} \mathrm{C}$. Electrophoresis was applied to confirm the formation of TET. The absorbance of MTX solution was detected by microplate reader to analyze the loading efficiency of MTX into TET. Fluorescence microscope was used to observe the intake of TET into cells. CCK8 experiment was applied to measure the vitality of cells. Flow cytometry was used to detect the apoptosis and polarization. CIA model was established based on DBA/1 mice. Mice were randomly divided into five groups: normal group injected with NS; after established CIA model, CIA group injected with NS, MTX group injected with MTX solution, MTX-TET group injected with MTX-TET NP. Results: We synthesized DNA tetrahedron(A) and used 8\% PAGE electrophoresis to confirmed the successfully synthesis(B). Then We found that when TET concentration fixed, the loading MTX concentration gradually increased and saturated at $190 \mu \mathrm{M}(C)$. While completely loading needed at least 4 hours(D). Fluorescence showed that single DNA strand cannot be taken by RAW, while TET can be easily taken by RAW(E). CCK8 showed that empty TET had no obvious effect on cells, while MTX and MTX-TET with equivalent concentration can obviously suppress the vitality $(F)$. Similarly, the apoptosis trial showed that TET can slightly decrease the apoptosis of RAW, MTX and MTX-TET can significantly promote the apoptosis $(\mathrm{G})$. Flow cytometry showed that the MTX-TET can decrease the expression of M1 marker CD80 $(\mathrm{H})$.
A

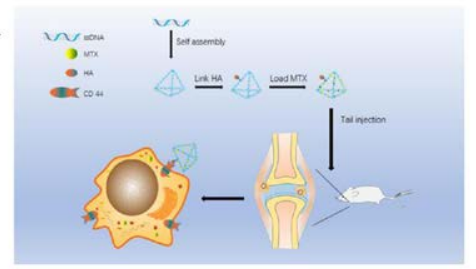

$\mathrm{C}$

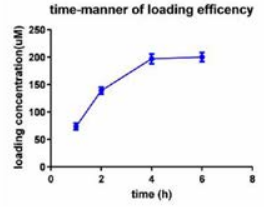

$\mathrm{E}$

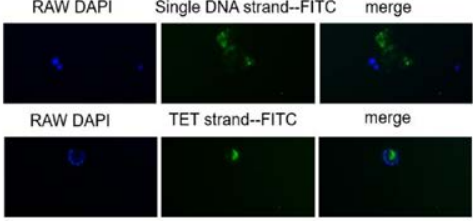

B

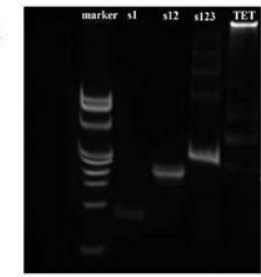

D

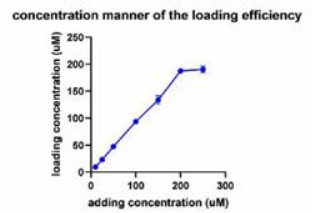

$\mathrm{H}$

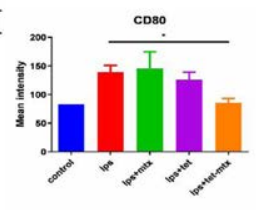

$\mathrm{F}$

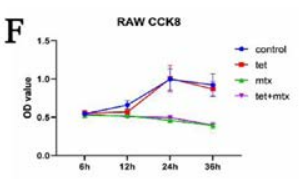

G

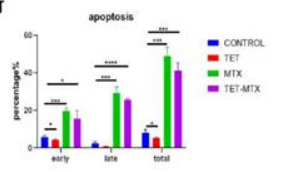

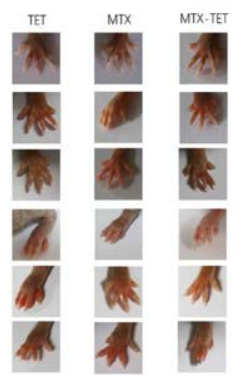

Figure 1.
At last, we treat mice with NS, TET, MTX and MTX-TET once a week after CIA model established, and found that TET have no significantly effect on mice, while MTX and MTX-TET can alleviate the inflammation symptom of paws(I).

Conclusion: Conclusions: We synthesized MTX-loading DNA tetrahedron conjugated with HA, and found that the MTX-TET NP have the excellent ability of promote RAW apoptosis and relieve proinflammatory M1 polarization. while also can alleviate the symptom of CIA mice.

REFERENCES:

[1] Aletaha D, Smolen JS: Diagnosis and Management of Rheumatoid Arthritis: A Review. JAMA 2018, 320(13):1360-1372.

[2] Smolen JS, Aletaha D, McInnes IB: Rheumatoid arthritis. Lancet 2016, 388(10055):2023-2038

[3] Hu Q, Li H, Wang L, Gu H, Fan C: DNA Nanotechnology-Enabled Drug Delivery Systems. Chem Rev 2019, 119(10):6459-6506.

Disclosure of Interests: None declared

DOI: 10.1136/annrheumdis-2021-eular.3690

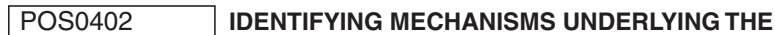 ASSOCIATION BETWEEN RHEUMATOID ARTHRITIS AND PERIODONTITIS USING IN SILICO ANALYSIS OF CANONICAL PATHWAYS}

K. S. K. Ma ${ }^{1}$, L. T. Wang ${ }^{2} .{ }^{1}$ National Taiwan University, Taipei, Taiwan, ROC, Department of Life Science, Taipei, Taiwan, Republic of China; ${ }^{2}$ National Taiwan University Hospital, Department of Obstetrics \& Gynecology, Taipei, Taiwan, Republic of China

Background: Periodontal pathogens such as Porphyromonas gingivalis (P.g.) has been proposed too involve in rheumatoid arthritis (RA) progression via citrullinating and producing exogenously citrullinated human and bacterial epitopes.

Objectives: We identified pathways downstream to periodontitis onset that involved in RA progression with RNA-sequencing data.

Methods: Canonical pathway analysis was conducted by comparing mRNA expression data from whole-transcriptome expression profiling using z-score and $p$-value visualization to identify underlying mechanisms among patients with RA $(n=7)$ and periodontitis $(n=9)$. Collected biopsies included human blood samples for RA and human gingival tissues for periodontitis. RNA-seq data with $-\log _{10}(P)$ values larger than 1.3 were considered significant, and positive $z$-scores indicated up-regulation. The statistical software was Ingenuity Pathway Analysis (QIAGEN).

Results: Among all significantly enriched $(-\log 10(P)>1.3)$ periodontitis-associated pathways underlying RA progression identified from the recruited cases, the production of nitric oxide and reactive oxygen species in macrophages, $\mathrm{B}$ cell receptor signaling, osteoarthritis pathway, HOTAIR regulatory pathway, IL-8 signaling, LPS/IL-1 mediated inhibition of RXR function, leukocyte extravasation signaling, and neuroinflammation signaling pathway, were up-regulated in RA patients and patients with periodontitis, when compared with patients without either disease. The $z$-scores of production of nitric oxide and reactive oxygen species in macrophages were 2.45 for RA $(-\log 10(P)=1.41), 3.89$ for periodontitis $(-\log 10(P)=4.25)$; the $z$-scores of $B$ cell receptor signaling were 2.44 for RA $(-\log 10(P)=1.93), 2.65$ for periodontitis $(-\log 10(P)=6.97)$; the $z$-scores of osteoarthritis pathway were 1.89 for RA $(-\log 10(P)=3.28), 2.33$ for periodontitis $(-\log 10(P)=4.31)$; the $z$-scores of HOTAIR regulatory pathway were 1.63 for RA $(-\log 10(P)=1.71), 3.40$ for periodontitis $(-\log 10(P)=3.68)$; the $z$-scores of IL-8 signaling were 1.34 for RA $(-\log 10(P)=1.30), 4.43$ for periodontitis $(-\log 10(P)=$ 6.05); the $z$-scores of LPS/IL-1 mediated inhibition of RXR function were 1.33 for RA $(-\log 10(P)=2.00), 1.27$ for periodontitis $(-\log 10(P)=3.44)$; and the $z$-scores of leukocyte extravasation signaling were 1.13 for RA $(-\log 10(P)=1.80)$, 3.68 for periodontitis $(-\log 10(P)=18.14)$.

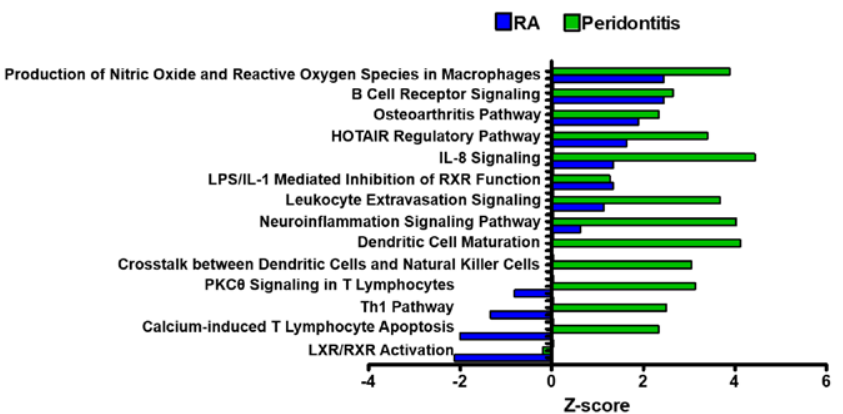

Figure 1. Canonical pathway analysis on transcriptome of blood samples for patients with RA and gingival tissues for periodontitis.

Conclusion: Our findings suggest that infection-driven activation of macrophages and $B$ cells is involved in RA pathogenesis. This occurs primarily 